\title{
The Analysis Research of Affected Factors of Spinning Force
}

\author{
Zhanjun Liu \\ Faculty of Aerospace Engineering,Shenyang Aerospace University,China \\ luzluzc@126.com
}

Keywords: spinning force; tensile strength; affected factors; deformation zone

\begin{abstract}
Spinning force is the spinning metal pipe tensile strength of a linear relationship. The higher is tensile strength, the bigger is the spinning force. For different kinds of material and heat treatment state, the deformation resistance is different, the required pressure is also different. Material usually make spinning force reduced after annealing. In spinning at constant external conditions, hot spinning also can lower the spinning force. Out some metal is under different temperature the tensile strength, the size of the spinning force mainly depends on the yield strength of material and contact area after the ball on the pipe pressure deformation zone. Thus deforming force is a function of metal processing of natural properties and external conditions.
\end{abstract}

\section{Introduction}

The ball spinning deforming is force characteristics. Ball spinning can be seen that the ball body is done as a tool for the tube billet extrusion forming, in the contact deformation zone, ball and the tube billet remain the state of rolling friction, and the deformation zone contacts with the deforming force. In the process of metal spinning process, the important parameters is deformation force in the process of spinning. It has to do with that spinning equipment can withstand the load, the working conditions of the process equipment, spinning processing[1].

Accuracy production efficiency has a close relationship. Therefore, grasps is the spinning force calculation and determination methods, such as, to increase productivity, reduce energy consumption, and promote science and technology progress, and to improve the tooling and equipment is very important. Metal and alloy plastic objects exist a kind of state. It not only depends on the object the nature of the internal and external conditions, also depends on the deformation. Such as stress deformation mode decided state, degree of deformation and temperature of the metal processed, etc., all have not suddenly on metal plastic visual impact.

\section{The Stress Deformation Area Is Analyzed}

Ball spinning, when plastic deformation of metal material is the ball of tube billet, is the rolling friction under implementation. Under the condition of sufficient lubrication, the pipe is less than between the ball and the friction coefficient 0.03 , which is far less than the average of the stamping die friction coefficient. To avoid a punching blade, it takes part with large parts of wall friction coefficient, thus ball spinning deformation is thin tube billet of smaller under axial tensile stress[2].

Because the ball spinning is defined as a plane strain state, the mean principal stress is equal to the critical half of the principal stress. Effect of pressure on the pipe wall thickness direction. Stress is normal maximum principal stress. From spinning tube billet is deformation zone. Distribution of force values of principal stress in the deformation zone is a variable. Axial force should be little value in deformation, which is a number of different factors. Not only from the qualitative aspect, but from the quantitative aspects. Study is all the factors that affect the deformation resistance, so as to seek the precise rules and deformation resistance.

It is theoretical calculation formula or method. In order to correctly select equipment, tools increases productivity and system of setting the reasonable technical process. For application of the theory, elastic-plastic deformation accurately describe the process. The complex is also very difficult. Therefore, in order to simplify the spinning force calculation in engineering, it is proposed to some basic assumptions[3]. 
Using appropriate mechanics principle makes the necessary mathematical calculation. In the project,the basic assumption is roughly as follows:

The deformation of tubing performance is homogeneous and isotropic, the pipe deformation does not change size, between the ball and tube billet friction, coefficient value is 0.03 , sometimes it can be ignored, in the process of spinning, equipment and mold is absolutely rigid, in the process of tube spinning, circumferential deformation is neglected, which holds that the deformation zone is viewed for plane deformation. To determine the deformation is plastic deformation of the object, the mechanical requirements must be met. Because in ball spinning, spinning deformation is usually the stress state, the deformation force and effect is on the ball without solid direction, therefore, the force is decomposed into three vertical component with each other.

Derivation and numerical simulation research are the relationship between the parameters of ball spinning technology cognitive play important role, on the analysis of the spinning, mechanics are borrowed from the extrusion forming area[4]. All pressure values, in fact, squeeze the fixed value of average pressure value to replace the ball spinning. Contact pressure value is not very accurate. Therefore, the author put forward by using plane strain condition.

To calculate the power parameters must first clear ball with fitting contact area, the author gives the space rectangular coordinate system of schematic diagram, the entire contact area is actually made up of three outlines spherica[5]1. The contact surface graphics can be reference for $3 \mathrm{~d}$ graphics. At the same time, it gives the ball spinning, the ball bearing contacts with the fitting of the three-dimensional diagram. In spinning process, the ball bearing of axial feed ratio is very small, single circle formed is almost negligible by the indentation force, so in order to simplify the calculation process, and by contact with contour line, besides the three lines is shown in removal of indentation added article, a circle with its center pipe is the intersection of vertical curve.

\section{Comparing Different Method Is to Calculate the Results}

Ball spinning process of spinning force is to determine the size of the spinning process parameters, to choose the ball diameter and determine the important basis of spinning equipment. Although the ball spinning process is narrow in industrial application scope, but simple superiority and particularity is of the process, also gradually cause widely attention in recent years. In order to calculate the ball spinning pressure and scholars at home and abroad, it provides a lot of calculation method and formula of spinning force. But due to the process parameters is of ball spinning of diversity, and plastic deformation mechanism is the complexity of calculation formulas in various assumptions, its borrow and simplify is inevitable, and it also makes the calculation accuracy affected. In industrial practice, however, it is mostly just a rough estimate the size of the spinning force, or ability to determine spinning equipment can meet the need of spinning process. In addition to reference for production practice experience data, the theoretical formula to calculate the spinning force data is more reliable. Some of the single ball spinning pressure calculation is formula for the actual operation, the each process parameter values are as follows: spinning materials is for nickel copper alloy, pipe diameter is of $20 \mathrm{~mm}$. The original wall thickness is finished by product wall thickness of $0.5 \mathrm{~mm}$, deformation before the yield limit is of $300 \mathrm{mpa}$, feeding is $0.1 \mathrm{~mm} / \mathrm{r}$, ball diameter is $10 \mathrm{~mm}$, ball number is 9 for the spinning deformation mode.

Through analysis and production practice, ball spinning pressure theory is of cognition, the size of the spinning force mainly depends on the yield strength of material and contact area after the ball on the pipe pressure deformation zone. Thus deforming force is a function of metal processing of natural properties and external conditions. Analysis of influence is factors and regularity of the spin, which further guides the spinning production and the theory research of ball spinning[6].

Spinning force is the spinning metal pipe tensile strength of a linear relationship. The higher is tensile strength, the bigger is the spinning force. For different kinds of material and heat treatment state, the deformation resistance is different, the required pressure is also different. Material usually make spinning force reduced 
after annealing. In spinning at constant external conditions, hot spinning also can lower the spinning force. Out some metal is under different temperature the tensile strength.

Wall thickness reduction ratio is a basic technological factors of affecting the rotary pressure. The ball bearing axial feeding affects on the spinning force.Thin ball spinning technology is usually used in special vertical spinning machine. Vertical cylinder piston rod is clamping sleeve on the mandrel pipe under uniform pressure slowly, and is placed in the spindle seat of internal model set of high speed rotation driving the ball around. Mould sleeve rotates the piston rod falling distance for spinning feed ratio, it is namely the feeding. After feeding, regardless of spiral pipe quality, spinning force is an important factor. With the increase of value, it directly affects the axial propulsion reduction, and spinning deformation speed increases. Whether positive rotation or reverse spin forms, the three spinning force components are also increased. Fig.1 shows curve of tangential force and feed ratio relation.
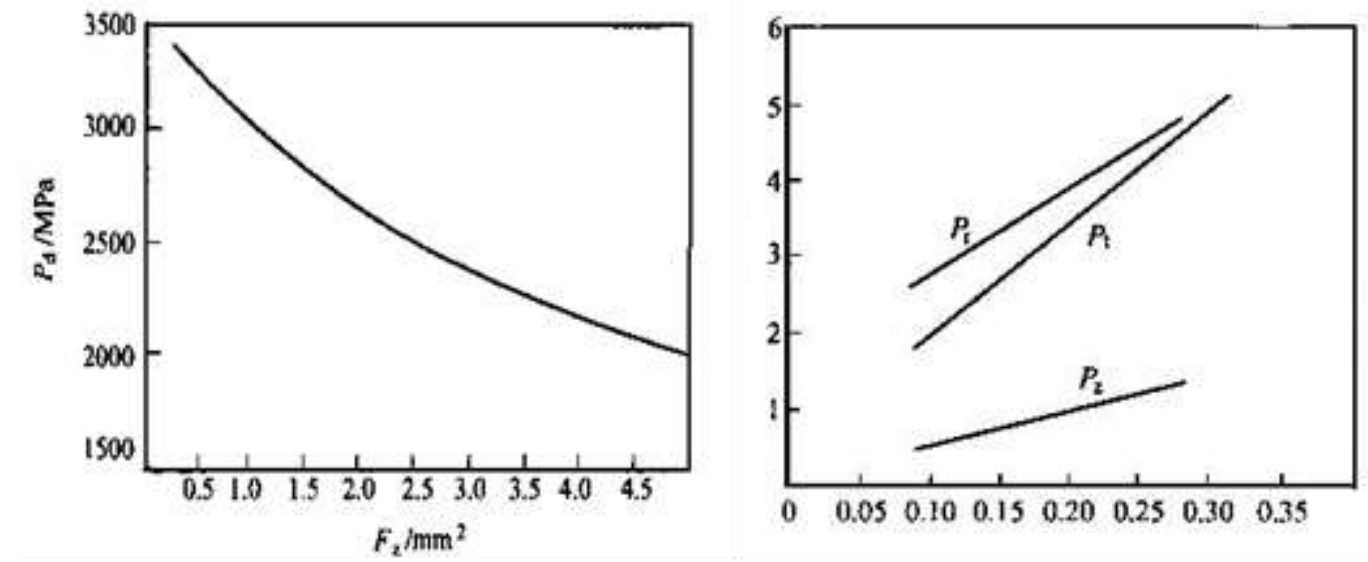

Figure 1. Curve of tangential force and feed ratio relation

There is reasonable spinning angle range. Angle of stress state is too big or too small, spinning and forming quality is not good. Because the characteristics of the ball change is the ball diameter, it is directly involved in the deformation of the factors. after the confirmation of the ball diameter, and quantity of thinning deformation contacts region arc, it also will determine the size of the ball. That spinning is proportional to the angle and ball diameter. This is ball spinning wheel spinning of cylindrical parts with different.

\section{The ball spinning deformation direction of the influence of the spinning force}

By the spinning force calculation formula of know, the size of the spinning force households is proportional to the ultimate strength and deformation of material. At the same time, also spinning processing is directly related to the changes of external conditions.

Ball spinning of tube billet are mostly metallurgy factory production, to provide hard products. Deformation of aluminum is after annealing. Reduce and increase of elongation value is advantageous to the spinning force. So sometimes in front of the spinning recrystallization annealing or solid solution hardening is very necessary.

Tubular and ball spinning is of cylindrical parts, due to the source of the billet, it often adopts different ways of spinning. With bottom of the barrel, it is used more positive spin, and tubular parts is with the method of reverse rotation. When spinning, metal material relative to the mandrel axis flows direction, there are two possible.

When positive rotation flows direction, and the spiral flows in the opposite direction. As shown in the positive rotation and reverse rotation of metal flow, and the influence of the friction resistance is different. The positive rotation is the metal flow direction and mandrel axial moving in the opposite direction. Reverse spin 
are when both the same, this will cause friction between the tube wall and the mandrel in a different direction, it will have different impact on the deformation force. Positive rotation of the friction is tensile stress, which is beneficial to the axial movement of the metal, the spinning force decreases.

At the same time, the deformation should be sought to know that the spin is three to the compressive stress. Positive rotation is second to the compressive stress in radial and circumferential axial tensile stress state. At this time of the tensile, stress on deformation area reduces the influence of deformation force. So the same external deformation conditions are spinning force, it is less than the spinning. Spinning process of the equipment and process have many other complex factors.

\section{Conclusions}

Spinning force is the spinning metal pipe tensile strength of a linear relationship. The higher is tensile strength, the bigger is the spinning force. For different kinds of material and heat treatment state, the deformation resistance is different, the required pressure is also different. Material usually make spinning force reduced after annealing. In spinning at constant external conditions, hot spinning also can lower the spinning force. Out some metal is under different temperature the tensile strength, the size of the spinning force mainly depends on the yield strength of material and contact area after the ball on the pipe pressure deformation zone. Thus deforming force is a function of metal processing of natural properties and external conditions.

\section{References}

[1] LiuZhanJun, Difficulty reliability prediction research of titanium plate stamping forming based on the fuzzy control, plastic engineering journal, Beijing, 2005, pp. 63-66.

[2] Feng. Jing, The Mixture of The Multimode System Reliability Growth Model,Computer Application Research Supplement, 2003, pp.60-61.

[3] Xu. Yuxiu, Complex Mechanical Fault Diagnosis Method of Fractal and Wavelet, Mechanical Industry Publishing House,Beijing, 2005, pp.24-25.

[4] Weng. chaoxi, Reliability Growth, Science Press, Beijing,1993, pp.130-131.

[5] Guofang, Reliability Data Collection And Analysis, National Defence Industry Press, 1995, pp. 22.

[6] Wen.WeiDong, Prediction model based on the fuzzy reliability, aircraft engine, Beijing,2003, pp.322-323. 